\title{
Correction to: Physical Activity, Sedentary Behavior, and Sleep before and after Bariatric Surgery and Associations with Weight Loss Outcome
}

\author{
Mette S. Nielsen ${ }^{1,2}$ (D) Hagir Alsaoodi ${ }^{1} \cdot$ Mads F. Hjorth $^{1}$ (D) Anders Sjödin ${ }^{1}$ \\ Published online: 2 December 2020 \\ (C) Springer Science+Business Media, LLC, part of Springer Nature 2020
}

\section{Correction to: Obesity Surgery.}

https://doi.org/10.1007/s11695-020-04908-3

In the original article there is a typographical error in the Purpose section of the Abstract. The corrected version follows:

Purpose Physical activity, sedentary behavior, and sleep have been linked to the likelihood of maintaining healthy body weight. This study aimed to determine objectively measured movement behaviors before and up to 18 months after bariatric surgery and to investigate whether preoperative levels of these movement behaviors and potential changes of these behaviors were associated with changes in body weight and body composition.

Publisher's Note Springer Nature remains neutral with regard to jurisdictional claims in published maps and institutional affiliations.

The online version of the original article can be found at https://doi.org/ $10.1007 / \mathrm{s} 11695-020-04908-3$

Mette S. Nielsen

msn@nexs.ku.dk

Hagir Alsaoodi

hagiir_91@hotmail.com

Mads F. Hjorth

madsfiil@nexs.ku.dk

Anders Sjödin

amsj@nexs.ku.dk

1 Department of Nutrition, Exercise and Sports, Faculty of Science, University of Copenhagen, Rolighedsvej 26, 1958 Frederiksberg

C, Denmark

2 The Danish Diabetes Academy, Søndre Blvd. 29, 5000 Odense, Denmark 